BULLETIN OF THE

AMERICAN MATHEMATICAL SOCIETY

Volume 78, Number 4, July 1972

\title{
CURVATURE FUNCTIONS FOR 2-MANIFOLDS WITH NEGATIVE EULER CHARACTERISTIC
}

BY JERRY L. KAZDAN ${ }^{1}$ AND F. W. WARNER ${ }^{1}$

Communicated by I. M. Singer, December 17, 1971

Introduction. In [4], [5], [6] we posed the problem of describing the set of Gaussian curvature functions $K$ that a given 2-manifold $M$ can possess. We observed that if $M$ is compact, the Gauss-Bonnet formula

$$
\int_{M} K d A=2 \pi \chi(M)
$$

imposes a sign condition on $K$. For example, $K$ must be negative somewhere if the Euler characteristic $\chi(M)$ is negative. We have asked if these sign conditions are sufficient conditions on a given function $K \in C^{\infty}(M)$ for $K$ to be the Gaussian curvature of some Riemannian metric on $M$.

To solve this problem, we have considered the more specific problem of attempting to realize $K$ as the curvature of a metric $\hat{g}$ pointwise conformal to a given metric $g$, so that $g=e^{2 u} g$ for some $u \in C^{\infty}(M)$. If $k$ is the curvature of the metric $g$, this becomes the problem of solving the nonlinear elliptic equation $[5, \S 5]$

$$
\Delta u=k-K e^{2 u}
$$

on $M$, where $\Delta$ is the Laplacian in the $g$ metric.

In our previous studies $([4],[5],[6])$ we have given a complete discussion of these questions if $\chi(M)=0$ as well as some partial results for $\chi(M) \neq 0$. We were also able to obtain information concerning the curvature functions of some open 2-manifolds. In this case there is no Gauss-Bonnet restriction, so one expects that any smooth function is the curvature of some Riemannian metric. A survey of related literature is given in [4], [5], [6] to which we refer the reader.

In this paper we completely settle the description of the set of Gaussian curvature functions on compact $M$ with $\chi(M)<0$ as well as the open manifold case for all open 2-manifolds obtained as "nicely punctured" compact manifolds. Thus this question remains open only in the case of $S^{2}$ for which partial results have been obtained in [3], [5], [11].

It turns out that the differential equation (1) becomes easier to work with if one frees it from the geometric situation and instead considers the more general equation $\Delta u=f+h e^{2 u}$ where $f$ and $h$ are prescribed functions, not tied to geometric considerations. In this paper we are concerned with

AMS 1970 subject classifications. Primary 35J25, 35J60, 53A90, 53C99; Secondary 35R05, 58G99, 47H15, 53C20.

${ }^{1}$ Supported in part by NSF Grants GP 28976X and GP 29258. 
the case in which $\int_{M} f<0$. By a suitable change of variables we can put this equation into the form

$$
\Delta u=-c+h e^{u}
$$

where $c$ is a positive constant. This is the form we work with. We might note that the techniques used for (2) to obtain the results here are entirely different from the calculus of variations techniques used in [5], [6]. The heart of our work concerns new existence and nonexistence theorems for (2). It is fascinating to see how the theory of (2) changes drastically depending on the sign of $c$.

In the next section we state the geometric results, while in the final section we state the facts concerning the differential equation (2) that are needed to prove the geometry results.

1. Let $M$ be a $C^{\infty}$ connected (not necessarily orientable) 2-dimensional manifold.

THEOREM 1. If $M$ is compact and $\chi(M)<0$, then $K \in C^{\infty}(M)$ is the Gaussian curvature of some Riemannian metric on $M$ if and only if $K\left(x_{0}\right)<0$ for some $x_{0} \in M$.

THEOREM 2. If $M$ is an open manifold obtained from a compact 2-manifold by removing a finite number of points, then every $K \in C^{\infty}(M)$ is the Gaussian curvature of some Riemannian metric on $M$.

REMARK. We can also prove Theorem 2 for some more general types of punctured compact manifolds. It would be nice to have the theorem in the case in which $M$ is obtained by removing an arbitrary closed set from a compact 2-manifold.

The basic result which yields Theorems 1 and 2 is the following concerning when $K$ is the curvature of a metric conformal to a given metric. Actually we prove a somewhat stronger version using only $L_{p}$ assumptions on $K$ for the application to Theorem 2 .

THEOREM 3. Consider (1) on a compact 2-manifold $M$ with $\chi(M)<0$ and $K \in C^{\infty}(M)$.

(a) A necessary condition for there to exist a solution of (1) is that $\int_{M} K e^{2 v} d A<0$, where $d A$ is the element of area of the metric $g$, and $\Delta v=k+c$, where $c=-\bar{k}=(-1 /$ area $M) \int_{M} k d A$.

(b) An even stronger necessary condition is that the unique solution of

$$
\Delta u-2 c u=2 K e^{2 v}
$$

must be positive.

(c) There exists $K \in C^{\infty}(M)$ with $\int_{M} K e^{2 v} d A<0$ for which (1) has no solution. 
(d) A sufficient condition for the existence of a solution of (1) is the existence of a solution of the differential inequality

$$
\Delta u \leqq k-K e^{2 u} .
$$

(e) Given any $K \in C^{\infty}(M)$ with $K\left(x_{0}\right)<0$ for some $x_{0} \in M$, there is a diffeomorphism $\varphi$ of $M$ such that there exists a solution of

$$
\Delta u \leqq k-(K \circ \varphi) e^{2 u} .
$$

2. In this section we present the basic facts concerning the equation (2). Here $M$, as before, is compact, connected, and not necessarily orientable. However, in this section the dimension of $M$ can be any integer $n \geqq 2$.

THEOREM 4. We consider equation (2) with $h \in C^{\infty}(M)$ a given function and with $c>0$.

(a) If a solution exists, then $\bar{h}=\left(\int h d V\right) /(\operatorname{Vol} M)>0$. Even more strongly, the unique solution of $\Delta u-c u=-h$ must be positive.

(b) If $\bar{h}>0$, then there is a constant $0<\gamma \leqq \infty$ depending on $h$ (which we write as $\gamma(h))$ such that one can solve (2) for all $0<c<\gamma(h)$, but cannot solve (2) if $c>\gamma(h)$.

In order to use this theorem one needs information on the constant $\gamma(h)$. This is supplied by the following theorem.

THEOREM 5. (a) $\gamma(h)=\infty$ if and only if $h(x) \geqq 0$ for all $x \in M$ but $h \equiv 0$.

(b) If $h_{1} \geqq h$, then $\gamma\left(h_{1}\right) \geqq \gamma(h)$. Also, $\gamma(h)=\gamma(\lambda h)$ for any constant $\lambda>0$.

(c) There is an $h$ with $\bar{h}>0$ such that $\gamma(h)<c$.

The key to proving Theorems 3(d), 4(b) and 5(b) is the next lemma. We need an $L_{p}$ version for the $L_{p}$ version of Theorem 3(d) which is needed in proving the geometric results (Theorem 2) for open manifolds. In what follows we will let $p$ denote some number with $p>n=\operatorname{dim} M$, and we will let $H_{s, p}, s \geqq 0$, denote the Sobolev space of functions all of whose derivatives of order $\leqq s$ are in $L_{p}(M)$.

MAIN Lemma. Given $h \in L_{p}(M)$, if there exist $u_{0}, v_{0} \in H_{2, p}$ such that

$$
\Delta u_{0}+c-h e^{u_{0}} \leqq 0, \quad \Delta v_{0}+c-h e^{v_{0}} \geqq 0 \quad \text { with } v_{0} \leqq u_{0},
$$

then there is a function $u \in H_{2, p}$ such that

$$
\Delta u+c-h e^{u}=0 .
$$

Moreover, $u$ is $C^{\infty}$ in any open set where $h$ is $C^{\infty}$.

The solution $u$ is obtained by the iteration procedure in [2, p. 370], modified to the $L_{p}$ situation. We have made use of the apparently old observation ([10], [ 1], [12], [13]) that sub and super solutions can be used to obtain crucial a priori bounds needed for the iterations. One can 
also use these bounds to apply the Schauder Fixed Point Theorem or Leray-Schauder degree theory and obtain a solution. We prefer the more constructive method of iteration.

In order to prove part of Theorem 5(a), one must show that if $h(x)<0$ in some open set, then $\gamma(h)<\infty$, that is, there is some $c>0$ such that one cannot solve (2). This follows from Theorem 4(a) and an easy result concerning operators in Banach spaces.

Asymptotic Lemma. Let $B_{1}, B_{2}$ be Banach spaces with $B_{2}$ a subspace of $B_{1}$ with the natural injection $B_{2} \hookrightarrow B_{1}$ continuous. Let $L: B_{2} \rightarrow B_{1}$ be a bounded linear map and assume that $(L-\alpha I): B_{2} \rightarrow B_{1}$ is invertible for all $\alpha \geqq 0$. Moreover, assume that

$$
\left\|(L-\alpha I)^{-1}\right\| \equiv \sup _{Z \in B_{1}}\left(\left\|(L-\alpha)^{-1} Z\right\|_{1} /\|Z\|_{1}\right) \leqq c(\alpha),
$$

where $c(\alpha) \rightarrow 0$ as $\alpha \rightarrow \infty$ and where \|\|$_{1}$ is the norm in $B_{1}$. If $Y \in B_{2}$ (not just $B_{1}$ ) let $X \in B_{2}$ be the unique solution of $L X-\alpha X=Y$. Then

$$
\lim _{\alpha \rightarrow \infty} \alpha X=-Y
$$

in the $B_{1}$ norm.

Corollary. If $\Delta \varphi-\lambda \varphi=f$ on $M$, then $\lim _{\lambda \rightarrow \infty} \lambda \varphi(x)=-f(x)$.

The methods used here can be generalized to apply to other equations with bad nonlinear terms (our $e^{u}$ ) as well as to Dirichlet and Neumann problems in bounded open sets in $R^{n}$. These results are new even in the ordinary differential equation case. Details will be developed in several forthcoming papers $([7],[9])$.

ADDED IN PROOF. The methods used here extend directly to the problem of which functions $K$, on a manifold $M$ of $\operatorname{dim} \geqq 3$, are scalar curvatures of some Riemannian metric. By again seeking this metric as pointwise conformal to a prescribed metric, one is led to another nonlinear elliptic equation. Our results are similar to those obtained here and in [5], [6]. In particular, we have new results on the pointwise conformal problem including an integrability condition similar to Theorem 3 of [5] providing an obstruction to solving the resulting equation on $S^{n}$ for arbitrary $K$. We can also show that on compact manifolds of $\operatorname{dim} \geqq 3$, any $C^{\infty}$ function which is negative somewhere is the scalar curvature of some Riemannian structure. This result cannot be improved without some topological assumptions for Lichnerowicz has shown that on a compact spin manifold with Hirzebruch $\hat{A}$ genus $\neq 0$ the scalar curvature of any metric is necessarily negative somewhere unless it is $\equiv 0$. On "nice" open manifolds of $\operatorname{dim} \geqq 3$ every $C^{\infty}$ function is a scalar curvature. Details will appear in $[8]$. 


\section{BIBLIOGRAPHY}

1. Donald S. Cohen, Multiple stable solutions of nonlinear boundary value problems arising in chemical reactor theory, SIAM J. Appl. Math. 20 (1971), 1-13.

2. R. Courant, Methods of mathematical physics. Vol. II: Partial differential equations, Interscience, New York, 1962. MR 25 \# 4216.

3. H. Gluck, The generalized Minkowski problem in differential geometry in the large, Ann. of Math. (to appear).

4. Jerry L. Kazdan and F. W. Warner, Integrability conditions for $\Delta u=k-K e^{2 u}$ with applications to Riemannian geometry, Bull. Amer. Math. Soc. 77 (1971), 819-823.

5. - Curvature functions for 2-manifolds. I (to appear).

6. - Curvature functions for 2-manifolds. II (to appear).

7. - Curvature functions for 2-manifolds. III. The negative Euler characteristic case (to appear).

8., Scalar curvature and conformal deformation of Riemannian structure (to appear).

9. - Remarks on some nonlinear elliptic equations (to appear).

10. M. A. Krasnoselskiǔ, Positive solutions of operator equations, P. Noordhoff Ltd., 1964 (translated from the 1960 Russian edition), see especially Chapter 4.

11. J. Moser, On a nonlinear problem in differential geometry (to appear).

12. D. H. Sattinger, Monotone methods in nonlinear elliptic and parabolic boundary value problems (to appear).

13. R. B. Simpson and Donald S. Cohen, Positive solutions of nonlinear elliptic eigenvalue problems, J. Math. Mech. 19 (1970), 895-910.

Department of Mathematics, University of Pennsylvania, Philadelphia, PennsylVANIA 19104 